\title{
Automatic correction of the time step in implicit simulations of thermomechanical problems
}

\author{
J.M.P. Martins ${ }^{1, a}$, D.M. Neto ${ }^{1}$, J.L. Alves ${ }^{2}$, M.C. Oliveira ${ }^{1}$ and L.F. Menezes ${ }^{1}$ \\ ${ }^{1}$ CEMUC, Department of Mechanical Engineering, University of Coimbra, Rua Luís Reis Santos, 3030-788 Coimbra, Portugal \\ ${ }^{2}$ CMEMS, Center for MicroElectroMechanics Systems, University of Minho, Campus de Azurém, 4800-058 Guimarães, Portugal
}

\begin{abstract}
The accurate numerical modelling of thermomechanical systems is important in several industrial applications. A new staggered coupling strategy is proposed to deal with thermo-elasto-plastic problems involving large deformations and rotations, which is combined with an automatic time-step control technique. The proposed strategy is based on the isothermal split approach. It differs from the classical scheme since, in each increment, there are two phases of interchange of information, instead of only one. In each increment, the solution procedure is divided in a prediction phase and a corrector phase, and the interchange of information is performed on both. The proposed strategy was implemented in the in-house quasi-static finite element code DD3IMP. Its performance is analysed and compared with the classical strategy and the iterative one, which are commonly employed for solving thermomechanical problems. The results indicate that the propose strategy contributes for improving the accuracy of the numerical solution, with a reasonable computational cost.
\end{abstract}

\section{Introduction}

The numerical modelling of thermomechanical systems is of high practical importance in many industrial applications. In fact, the prediction of temperature and stress fields (considering its reciprocal effects) is a key factor in various manufacturing processes, e.g. in the warm sheet metal forming. The interdependence between these two fields manifests itself through heat generation by plastic deformation and, consequently, by temperature increase and softening of the sheet material.

The finite element method has become a standard tool for the analysis of these problems. Nevertheless, the development and implementation of numerical models to deal with thermomechanical problems is a challenge, since the interdependence between thermal and mechanical fields requires an efficient coupling strategy. Intensive research has been conducted on this subject during the last years. Typically, the treatment of thermomechanical problems through the finite element method can be carried out using two different coupling strategies: (i) the monolithic strategy or (ii) the staggered strategy $[1,2]$. The monolithic strategy deals with both fields (temperature and stress) simultaneously within each increment, providing a single system of equations with all variables involved. The main advantage is the unconditional stability. Nevertheless, this strategy is rarely used because the resulting system of equations is very large and can be nonsymmetric, depending on the formulation adopted. These characteristics result in a large computational cost [3-5]. Accordingly, the staggered strategy has been developed to reduce this cost. In this strategy, the two fields are treated

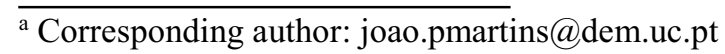

separately, yielding two system of equations. This allows to take advantage of the usage of different time scales and different spatial discretization methods for each field [3]. Moreover, the implementation of this coupling strategy in the existing finite element codes is considerably easier [6].

The staggered strategy can be employed following two different approaches, which differ between them in the stability. The standard approach is called isothermal split, where the global thermomechanical problem is split into a mechanical problem, which is solved at a constant temperature, and a heat conduction problem, which is solved at a fixed configuration [7]. The drawback of this approach is the conditional stability, which arises when the thermoelastic effects play an important role. However, unstable approximations only occur for values of the thermal expansion coefficient considerably higher than the ones found in metals [3]. Therefore, several authors have adopted this approach to solve metal forming problems (e.g. [8-10]). In order to overcome the conditional stability of the isothermal split, Armero and Simo [11] proposed another approach, the so-called isentropic split (also referred as adiabatic split), which can retain the property of unconditional stability. In the isentropic split, the mechanical problem is solved at a constant entropy, followed by the heat conduction problem solved at a fixed configuration. This approach is usually adopted in works based on hyperelastic formulations and multiplicative decomposition of the deformation gradient (e.g. [12-14]).

The staggered strategy combined with the isothermal split approach is the one adopted in the present study. This strategy was implemented in the in-house quasi-static 
finite element code DD3IMP [15,16], which was specially developed to simulate the sheet metal forming process. The main characteristics of this finite element code are the adoption of a hypoelastic law for the material behaviour description, while the evolution of the deformation process is described by an update Lagrangian formulation. Regarding the algorithm used for solving the mechanical problem, an explicit approach is used to determine a trial solution for the nodal displacements. After that, in order to improve the convergence rate of the iterative NewtonRaphson algorithm, used to correct the trial solution, an automatic time-step control technique is employed to restrict the increment size, called $r_{\text {min }}$ strategy $[17,18]$.

This study focuses the thermo-elasto-plastic problem with large strains and rotations, neglecting the contact with friction conditions, although these are also important in the modelling of forming processes [19]. The outline of this paper is as follows. A brief introduction of the mechanical model formulation is presented in section 2 . In section 3 , the equation of heat conduction and the numerical methods used for its resolution are presented. The detailed description of the proposed thermomechanical coupling algorithm is presented in section 4 . Section 5 contains two numerical examples, aiming to validate the numerical implementation and to assess the performance of the different coupling algorithms tested in terms of accuracy. The last section contains the concluding remarks.

\section{Mechanical Model}

\subsection{Large deformation continuum mechanics}

Let $\mathcal{B}$ be a continuum body, which consist of a set of particles defined by spatial points embedded on the domain $\Omega_{0} \in \mathbb{R}^{3}$. At the time instant $t_{0}$, the continuum body assumes the configuration $C_{0}$, called reference configuration, where the position of a generic point is defined by the position vector $\mathbf{x}_{0}$. After the application of some external stimuli, the continuum body assumes another configuration $C$, called current configuration, at the time instant $t$, where the same point of the reference configuration can be defined by the position vector $\mathbf{X}$. Thus, the evolution of each point with time is defined by a deformation mapping, according to

$$
\mathbf{x}=\mathbf{x}\left(\mathbf{x}_{0}, t\right) .
$$

The displacement of the spatial point, $\mathbf{x}_{0}$, between the reference and current configuration, can be defined by

$$
\mathbf{u}=\mathbf{x}\left(\mathbf{x}_{0}, t\right)-\mathbf{x}_{0},
$$

and its velocity by

$$
\mathbf{v}=\dot{\mathbf{x}}=\frac{\partial \mathbf{x}\left(\mathbf{x}_{0}, t\right)}{\partial t} .
$$

The deformation gradient, $\mathbf{F}$, defined for the current configuration $C$, is

$$
\mathbf{F}=\frac{\partial \mathbf{x}}{\partial \mathbf{x}_{0}} \quad \text { with } \operatorname{det} \mathbf{F}>0
$$

and the corresponding velocity gradient is given by

$$
\mathbf{L}=\frac{\partial \mathbf{v}}{\partial \mathbf{x}}=\dot{\mathbf{F F}} \mathbf{F}^{-1}
$$

which can be decomposed in a symmetric and an antisymmetric part. The symmetric part is called the strain rate tensor, $\mathbf{D}$, and the antisymmetric part, is the spin rate tensor, $\mathbf{W}$. These can be defined as

$$
\begin{aligned}
& \mathbf{D}=\frac{1}{2}\left(\mathbf{L}+\mathbf{L}^{\mathrm{T}}\right), \\
& \mathbf{W}=\frac{1}{2}\left(\mathbf{L}-\mathbf{L}^{\mathrm{T}}\right) .
\end{aligned}
$$

\subsection{Constitutive model}

A hypoelastic stress-strain relation is adopted for the description of the material behaviour. In this case, the strain rate tensor can be addictively decomposed as $[9,20,21]$

$$
\mathbf{D}=\mathbf{D}^{\mathrm{e}}+\mathbf{D}^{\text {th }}+\mathbf{D}^{\mathrm{p}},
$$

where $\mathbf{D}^{\mathrm{e}}, \mathbf{D}^{\text {th }}$ and $\mathbf{D}^{\mathrm{p}}$ are the elastic, the thermal and the inelastic (plastic) strain rate contributions, respectively. Thus, for describing a thermo-elasto-plastic material, the hypoelastic relation, which relates the strain rate with the stress rate, can be expressed as

$$
\dot{\boldsymbol{\sigma}}^{\mathrm{J}}=\mathbf{C}^{\mathrm{ep}}:\left(\mathbf{D}-\mathbf{D}^{\mathrm{th}}-\mathbf{D}^{\mathrm{p}}\right)
$$

where $\dot{\boldsymbol{\sigma}}^{\mathrm{J}}$ is the Jaumann derivative of the Cauchy stress tensor $\boldsymbol{\sigma}$, which is defined by

$$
\dot{\boldsymbol{\sigma}}^{\mathrm{J}}=\dot{\boldsymbol{\sigma}}+\boldsymbol{\sigma} \mathbf{W}-\mathbf{W} \boldsymbol{\sigma} .
$$

The $\mathbf{C}^{\text {ep }}$ in (9) is the elastoplastic module, which is a fourth-order tensor. The expression for this tensor depends of the algorithms adopted in the integration of the constitutive model and the type of relation considered between the states at the beginning and at the end of the loading increment. Thus, it is possible to consider the tangent elastoplastic modulus or the consistent elastoplastic modulus.

\subsubsection{Thermo-elasto-plastic state}

The associated flow rule is adopted, which implies that the plastic deformation rate tensor, $\mathbf{D}^{\mathrm{p}}$, is given by the relation

$$
\mathbf{D}^{\mathrm{p}}=\dot{\lambda} \frac{\partial f(\bar{\sigma}, Y)}{\partial \boldsymbol{\sigma}}
$$

where $\dot{\lambda}$ is designated as the plastic multiplier, which can be determined by the so-called consistency condition i.e. 
$\dot{f}=0$. The scalar function $f$ defines the yield surface and can be formulated as

$$
f(\bar{\sigma}, Y)=\bar{\sigma}-Y=0
$$

where $Y$ denotes the flow stress in simple tension, while $\bar{\sigma}$ represents the equivalent stress, which for von Mises yield criterion is given by

$$
\bar{\sigma}=\sqrt{\frac{3}{2} \boldsymbol{\sigma}^{\prime}: \boldsymbol{\sigma}^{\prime}}
$$

where $\boldsymbol{\sigma}^{\prime}$ is the Cauchy deviatoric stress tensor.

The evolution of the flow stress is a combination of linear and saturation hardening [3], given by

$$
Y\left(\bar{\varepsilon}^{\mathrm{p}}, T\right)=h \bar{\varepsilon}^{\mathrm{p}}+\left(Y_{\infty}-Y_{0}\right)\left(1-\exp \left(-\delta \bar{\varepsilon}^{\mathrm{p}}\right)\right),
$$

where $h, Y_{0}$ and $Y_{\infty}$ are the linear hardening modulus, initial yield stress and saturation stress, respectively. Besides, they depend on temperature by the following relations

$$
\begin{gathered}
h(T)=h_{0}\left[1-\omega_{h}\left(T-T_{0}\right)\right], \\
Y_{0}(T)=Y_{0}\left[1-\omega_{0}\left(T-T_{0}\right)\right], \\
Y_{\infty}(T)=Y_{\infty}\left[1-\omega_{h}\left(T-T_{0}\right)\right] .
\end{gathered}
$$

where $T_{0}$ is the reference temperature and $\omega_{h}$ and $\omega_{0}$ are the parameters for the description of thermal softening. The description of the flow stress behaviour is completed with the saturation exponent $\delta$.

\subsubsection{Thermal strain rate}

The thermal strain rate tensor involved in (8) can be written as

$$
\begin{gathered}
\mathbf{D}^{\text {th }}=\alpha_{\mathrm{T}}^{*} \dot{T} \mathbf{I}, \\
\alpha_{\mathrm{T}}^{*}=\alpha_{\mathrm{T}}+\frac{\partial \alpha_{\mathrm{T}}}{\partial T}\left(T-T_{0}\right),
\end{gathered}
$$

where $\alpha_{\mathrm{T}}$ is the thermal expansion coefficient, $T$ and $\dot{T}$ are the temperature and the temperature rate, respectively, while I is the second-order identity tensor.

\subsection{Principle of virtual velocities}

For the reference configuration, the region occupied by the deformable body is $\Omega_{0} \in \mathbb{R}^{3}$ and its boundary is $\Sigma_{0}$. The boundary can be decomposed in two complementary parts: $\Sigma_{0 \mid 1}$, where the nominal stress vector is assigned, and $\Sigma_{0 \mid 2}$, where the velocity vector is prescribed. For this reference configuration, in an update Lagrangian scheme, the principle of virtual velocities is given by:

$$
\int_{\Omega_{0}} \mathbf{S}: \delta \dot{\mathbf{F}} \mathrm{d} \Omega=\int_{\Sigma_{0 \mid 1}} \mathbf{s} \cdot \delta \mathbf{v} \mathrm{d} \Sigma
$$

which has to be valid for any virtual velocity field $\delta \mathbf{v}$ (with $\delta \mathbf{v}=0$ on the boundary $\Sigma_{0 \mid 2}$ ). $\mathbf{S}$ is the first PiolaKirchhoff stress tensor, $\dot{\mathbf{F}}$ is the time derivative of the deformation gradient and $\mathbf{s}$ corresponds to the external loading, i.e. the prescribed nominal stress vector.

\section{Thermal model}

The differential equation governing the heat conduction within a continuum body $\mathcal{B}$, defined by the open set $\Omega \in \mathbb{R}^{3}$ for the current configuration, can be derived from the first principle of thermodynamics, which combined with the Fourier's heat conduction law, assumes the following form:

$$
\rho c \frac{\partial T}{\partial t}=\operatorname{div}(k \cdot \operatorname{grad}(T))+\dot{W}^{\mathrm{pl}}+\dot{q},
$$

where $\rho$ is the density, $c$ is the specific heat, $T$ is the temperature, $k$ is the thermal conductivity, $\dot{W}^{\mathrm{pl}}$ is the internal heat generation term due to plastic deformation, and $\dot{q}$ corresponds to an heat source. This last term does not play any role in this work, thus it is neglected. Furthermore, the thermo-elastic coupling effect is not considered, since its effect is typically small, when compared with the one associated with heating induced by plastic deformation.

The heat generated by plastic deformation term, $\dot{W}^{\mathrm{pl}}$, usually involved in the modelling of thermomechanical problems, is the fraction of plastic power converted into heat. The remaining part of plastic power, which is not converted into heat power, is stored in the material [22]. This effect is often modelled using the assumption of Taylor and Quinney [23], which states that 5\% to $15 \%$ of the plastic work is stored in the material. Thus, the heat generated by plastic deformation is expressed by

$$
\dot{W}^{\mathrm{pl}}=\chi \boldsymbol{\sigma}: \mathbf{D}^{\mathrm{p}}
$$

where the parameter $\chi$ is called the Taylor-Quinney coefficient, which varies between 0.85 and 0.95 .

The thermal boundary conditions, such as convection, radiation and heat conduction by thermal contact, are important aspects to be considered in numerical models of thermomechanical problems $[24,25]$. Nevertheless, they are not addressed in this study, since they can be neglected in the comparison of staggered algorithms performance.

Applying the principle of virtual temperature and proceeding to the decomposition of the domain, the initial boundary value problem defined in (21) can be given in the matrix form as [26]:

$$
\mathbf{C} \dot{\mathbf{T}}+\mathbf{K T}=\mathbf{f},
$$

where the matrixes and vector are given by

$$
\begin{aligned}
\mathbf{C} & =\int_{\Omega} \rho c \mathbf{N}^{\mathrm{T}} \mathbf{N} \mathrm{d} \Omega, \\
\mathbf{K} & =\int_{\Omega} k \mathbf{M}^{\mathrm{T}} \mathbf{M} \mathrm{d} \Omega, \\
\mathbf{f} & =\int_{\Omega} \mathbf{N}^{\mathrm{T}} \dot{W}^{\mathrm{pl}} \mathrm{d} \Omega,
\end{aligned}
$$


where $\mathbf{N}$ is the vector of shape functions and $\mathbf{M}=\operatorname{grad}(\mathbf{N})$. The consistent form of the capacitance matrix $\mathbf{C}$ is replaced by the diagonal form, which avoids unstable results, incoherent with physical reality, and has the advantage of reducing the computational cost.

The numerical solution of the transient heat conduction equation (23) can be obtained throughout the adoption of the different numerical methods. Typically, the so-called $\alpha$-method is adopted for time integration. This method is given by the following assumptions [26,27]:

$$
\begin{gathered}
\dot{\mathbf{T}}_{t+\alpha \Delta t}=\left(\mathbf{T}_{t+\Delta t}-\mathbf{T}_{t}\right) / \Delta t, \\
\mathbf{T}_{t+\alpha \Delta t}=(1-\alpha) \mathbf{T}_{t}+\alpha \mathbf{T}_{t+\Delta t},
\end{gathered}
$$

where the parameter $\alpha$ can vary between 0 and 1 , which gives rises to different time integration methods, such as, explicit Euler forward ( $\alpha=0)$, Crank-Nicolson ( $\alpha=1 / 2$ ), Galerkin $(\alpha=3 / 2)$ and implicit Euler backward $(\alpha=1)$.

\section{Thermomechanical coupling}

This section presents a description of the proposed thermomechanical coupling algorithm, as well as the technique used for the automatic time step control, called $r_{\text {min }}$ strategy. The proposed algorithm is compared with the classical staggered algorithms, highlighting its advantages and drawbacks.

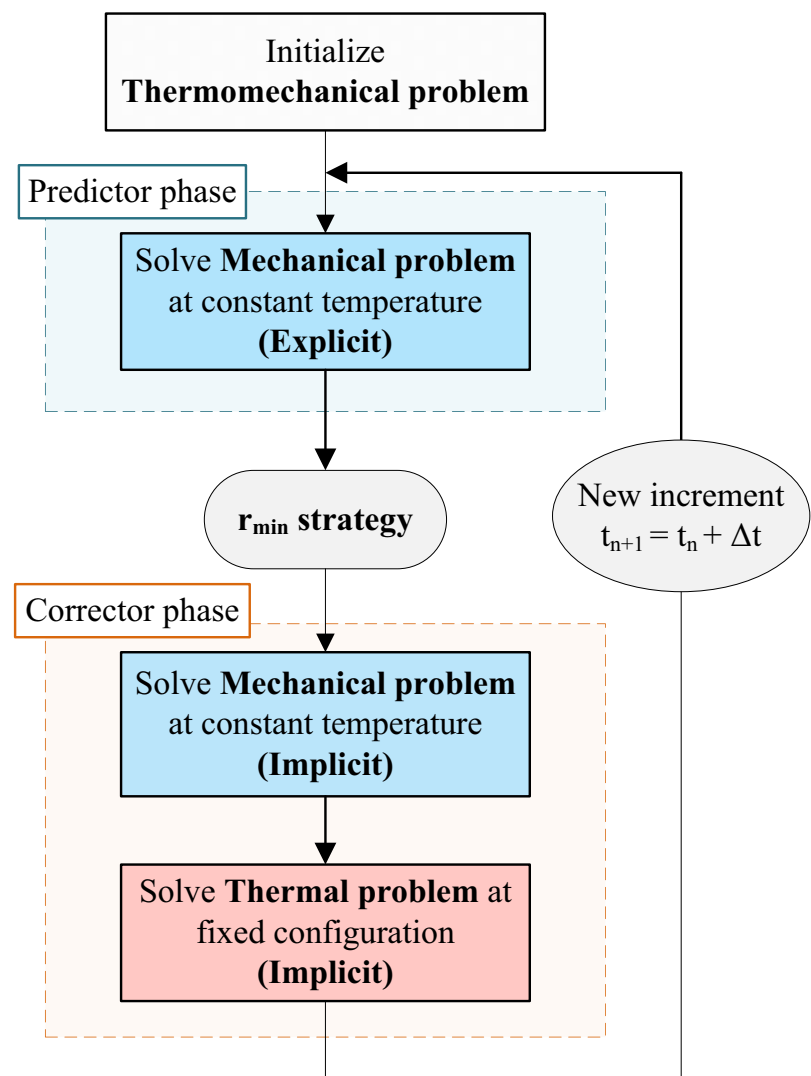

Figure 1. Classical coupling algorithm denoted by MMT.

The widely used classical staggered algorithm, comprises the interchange of information between the two fields only once, at each time increment. A diagram of this strategy, adapted to accommodate the $r_{\min }$ strategy, is presented in Figure 1. Since the mechanical problem in the corrector phase is solved using the temperature of the previous increment, there is always a delay between the two fields, which induces a loss of accuracy. On the other hand, the computational cost is relatively small due to the sequential solution of the mechanical and thermal problems. In order to improve the accuracy of the numerical solution, another coupling strategy has been developed, usually called iterative coupling algorithm [4]. In this case, the interchange of information between the two fields is performed through an iterative procedure, aiming to reach the fully converged solution. The mechanical and thermal problems are solved in the same sequence as presented in Figure 1, but the procedure is repeated, within each time increment, until attaining the convergence criterion. Consequently, higher computational cost is involved, due to the iterative cycle within each increment.

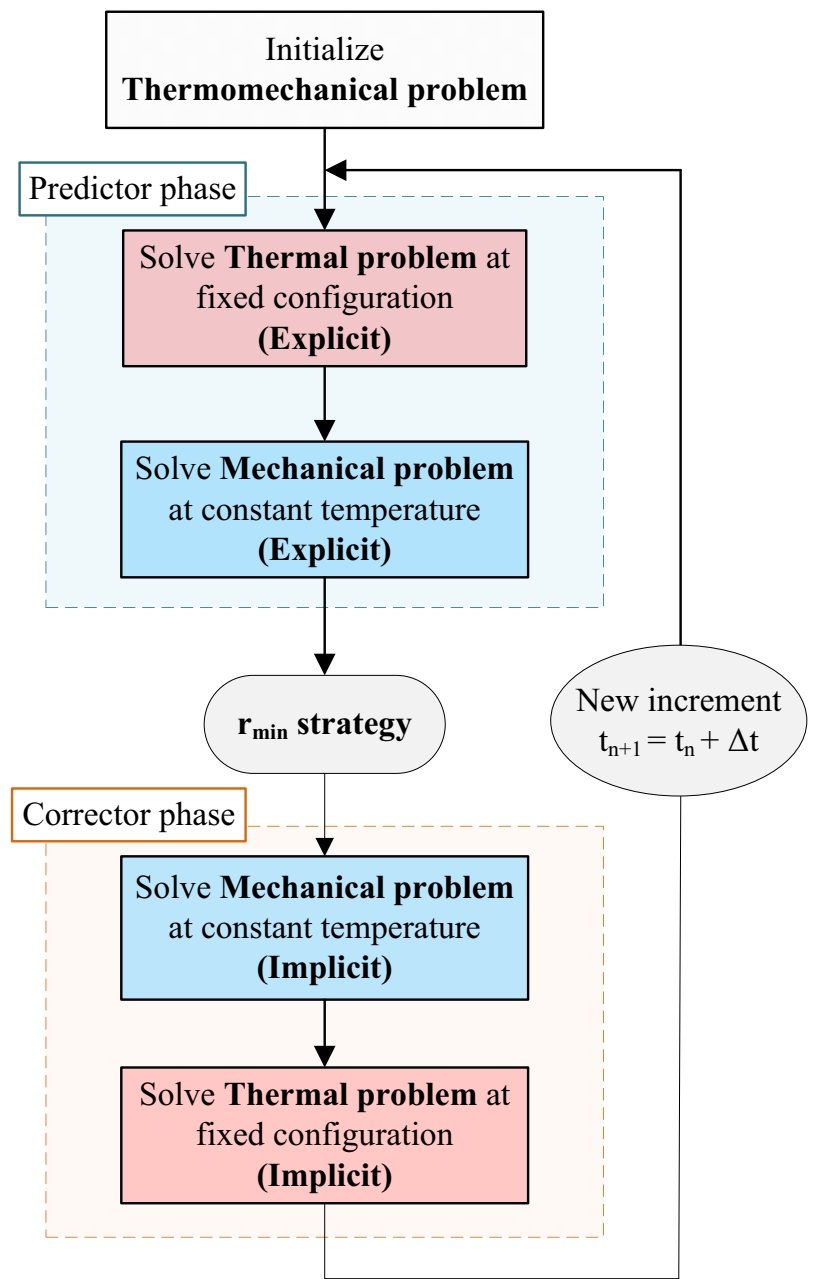

Figure 2. Proposed algorithm for the thermomechanical coupling, denoted by TMMT.

The proposed coupling algorithm was developed to reach a better compromise between computational cost and accuracy of results. The main difference to the classical staggered algorithms is the way the information between the two fields is interchanged. The proposed algorithm can be clearly divided in two phases, a prediction phase and a corrector phase, as shown in Figure 2. The first phase comprises solving the thermal problem 
for a user-defined time step, adopting an explicit or semiimplicit approach (depending on the value defined for the parameter $\alpha$ in (28)). In this first phase, the amount of heat generated by plastic deformation is assumed to be equal to the one of the last time increment. Thus, the result is a trial temperature field. After that, the mechanical problem is solved for this trial temperature field, adopting an explicit approach. At the end of this prediction phase, there is a trial displacement field and a trial temperature field. Since the integration methods adopted are explicit, the calculated variables are proportional to the time increment. Thus, in order to assure the convergence of the subsequent corrector phase, the $r_{\min }$ strategy is applied. This strategy adjusts the increment size according to some restrictions defined for the rotation, strain and stress increment. Based on that, it is possible to determine a weighting factor $r$, which is used to calculate a time increment that is expected to avoid convergence problems. The same weighting factor is used to calculate the new displacement field and temperature field, based on the trial solutions, since these are proportional to the time increment. More details about the application of this strategy to the mechanical problem can be found in Oliveira and Menezes [18]. Afterwards, the corrector phase is performed, where the mechanical problem is solved first, using the trial temperature field. The nonlinear system of equations resultant from the large thermoelasto-plastic deformation problem is solved with a Newton-Raphson iterative method. Thus, the previous displacement field, which corresponds to the initial solution for the Newton-Raphson method, is corrected until the equilibrium state is attained. Finally, the thermal problem is solved using an implicit approach (with the parameter $\alpha=1$ in (28)), for the current equilibrium configuration.

\section{Numerical examples}

\subsection{Expansion of a thick-walled cylinder}

The purpose of this example is to validate the developed finite element code on the resolution of thermomechanical problems. This validation was carried out using the iterative coupling algorithm to assure the most accurate results. The example considered deals with the radial expansion of thick-walled infinite cylinder, which was proposed by Argyris and Doltsinis [28] and later adapted by Simo and Miehe [29].

A thick-walled cylinder with an inner radius of $100 \mathrm{~mm}$ and an outer radius of $200 \mathrm{~mm}$ is subjected to an internal pressure. In the presented model, the internal pressure is replaced by an imposed radial displacement of the inner surface, with a magnitude of $130 \mathrm{~mm}$. The cylinder is assumed infinite long, along the axial direction, which allows the use of plain strain conditions in this direction. The thermal problem is solved assuming the external surface of the cylinder adiabatic and the only source of heat is the internal heat generated by plastic deformation. The cylinder as an initial temperature of $293 \mathrm{~K}$.

The material parameters are the ones proposed by Simo and Miehe [29] and are listed in Table 1. Only one quarter of the cylinder was modelled, imposing symmetry conditions. The mesh was composed by linear hexahedral finite elements, using 10 elements through the cylinder thickness and 10 elements along the hoop direction. A selective reduced integration technique was adopted for the mechanical problem while for the thermal problem full integration was applied. The time step discretization was automatically adapted throughout the $r_{\min }$ strategy.

Table 1. Material properties of the cylinder.

\begin{tabular}{|l|l|}
\hline Young' modulus & $E=70000 \mathrm{GPa}$ \\
\hline Poisson's ratio & $v=0.3$ \\
\hline Initial yield stress & $Y_{0}=70 \mathrm{MPa}$ \\
\hline Isotropic hardening modulus & $h=210 \mathrm{MPa}$ \\
\hline Thermal expansion coef. & $\alpha_{\mathrm{T}}=23.8 \times 10^{-6} \mathrm{~K}^{-1}$ \\
\hline Conductivity & $k=150 \mathrm{Wm}^{-1} \mathrm{~K}^{-1}$ \\
\hline Density & $\rho=2900 \mathrm{~kg} \mathrm{~m}^{-3}$ \\
\hline Heat capacity & $c=900 \mathrm{~J} \mathrm{~kg}^{-1} \mathrm{~K}^{-1}$ \\
\hline Taylor-Quinney factor & $\chi=0.9$ \\
\hline Thermal softening & $\omega_{0}=3.0 \times 10^{-4} \mathrm{~K}^{-1}$ \\
\hline Hardening/softening & $\omega_{h}=0 \mathrm{~K}^{-1}$ \\
\hline Reference temperature & $T_{\text {ref }}=293 \mathrm{~K}$ \\
\hline
\end{tabular}

Figure 3 shows the results obtained for the particular case of an adiabatic loading, i.e. since the internal conduction is neglected it corresponds to a loading duration $t \rightarrow 0 \mathrm{~s}$. Both the internal pressure evolution and temperature evolution are in good agreement with the numerical results obtained by Simo and Miehe [29].

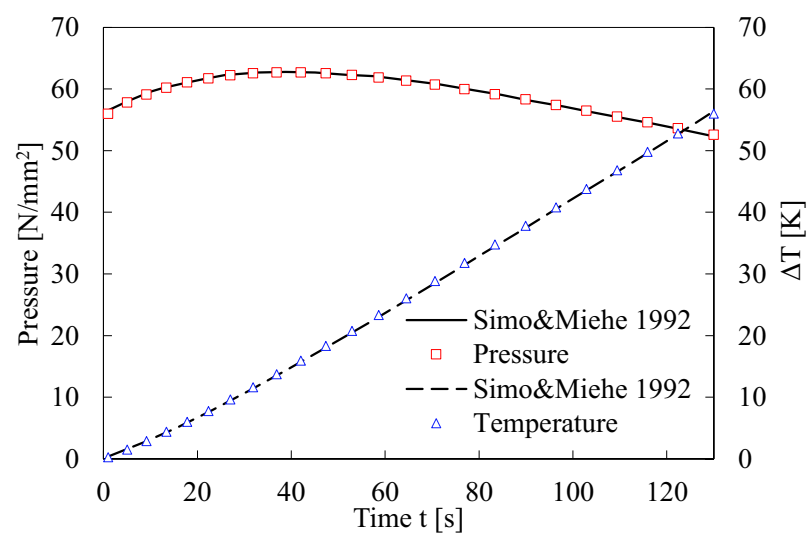

Figure 3. Evolution of the internal pressure and relative temperature $\left(\Delta \mathrm{T}=\mathrm{T}_{\mathrm{i}}-\mathrm{T}_{\mathrm{ref}}\right)$ at the inner surface of the cylinder for the adiabatic load case.

Figure 4 shows the temperature distribution along the radial position, considering different durations for the test, such as $t \rightarrow 0,1.3,13130 \mathrm{~s}$ and $\rightarrow \infty$. The increase in the total time enables a more homogeneous temperature distribution, resultant from the heat conduction within the material. 


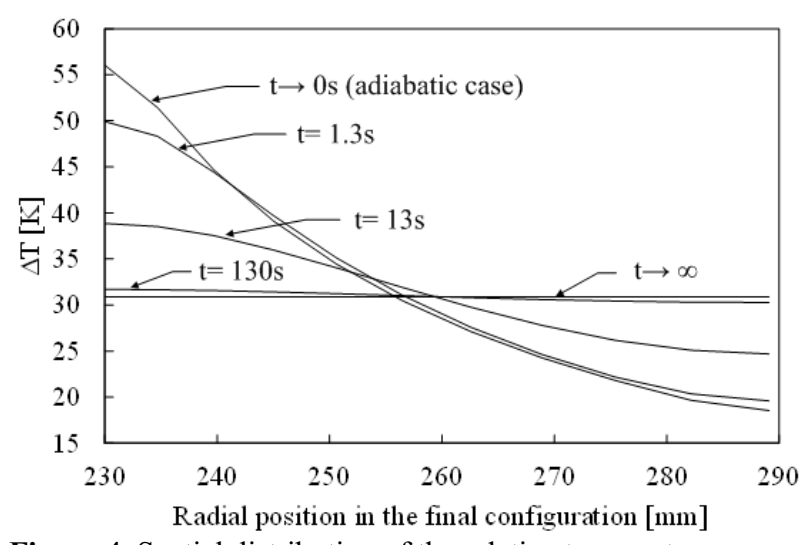

Figure 4. Spatial distribution of the relative temperature $\left(\Delta \mathrm{T}=\mathrm{T}_{\mathrm{i}}-\mathrm{T}_{\mathrm{ref}}\right)$ for the final configuration (radial displacement of $130 \mathrm{~mm}$ ) for different values of total time.

\subsection{Single element tensile test}

The objective of this example is to assess the performance of the proposed staggered algorithm in terms of accuracy. Therefore, it is compared with the two classical algorithms described in section 4. In order to improve the results discussion, on a first, the test was performed with the $r_{\text {min }}$ strategy deactivated. Afterwards, the tests were performed with this strategy activated. Thus, the impact of the $r_{\text {min }}$ strategy can be evaluated separately. For the sake of clarity the following nomenclature is adopted: the proposed algorithm is denoted by TMMT, the algorithm presented in Figure 1 is denoted by MMT and the iterative algorithm is denoted by MMIT.

The example consists on a single finite element (linear hexahedral element) with dimensions $1 \times 1 \times 1 \mathrm{~mm}^{3}$, which is submitted to a tensile stress state, by imposing a displacement $u=2 \mathrm{~mm}$ in one direction, at a constant velocity $v=1 \mathrm{~mm} \mathrm{~s}^{-1}$. The configuration of the single element is schematically represented in Figure 5. As for the previous example, selective reduced integration is considered for the mechanical problem and full integration for the thermal problem. The boundaries of the element are considered isolated from the environment and the only heat source is the heat generated by plastic deformation. The initial temperature was defined as $293 \mathrm{~K}$. The material parameters used in this example are listed in Table 2.

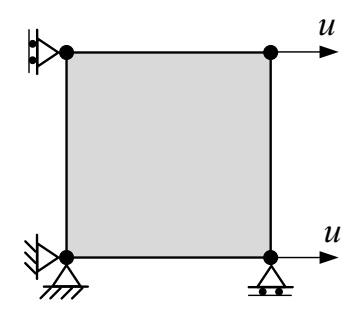

Figure 5. Configuration used for the single element test.
Table 2. Material properties used in the single element test.

\begin{tabular}{|l|l|}
\hline Young' modulus & $E=206900 \mathrm{GPa}$ \\
\hline Poisson's ratio & $v=0.29$ \\
\hline Initial yield stress & $Y_{0}=450 \mathrm{MPa}$ \\
\hline Isotropic hardening modulus & $h=129.24 \mathrm{MPa}$ \\
\hline Thermal expansion coef. & $\alpha_{\mathrm{T}}=1.0 \times 10^{-5} \mathrm{~K}^{-1}$ \\
\hline Conductivity & $k=45 \mathrm{Wm}^{-1} \mathrm{~K}^{-1}$ \\
\hline Density & $\rho=7800 \mathrm{~kg} \mathrm{~m}^{-3}$ \\
\hline Heat capacity & $c=460 \mathrm{~J} \mathrm{~kg}^{-1} \mathrm{~K}^{-1}$ \\
\hline Taylor-Quinney factor & $\chi=0.9$ \\
\hline Thermal softening & $\omega_{0}=2.0 \times 10^{-3} \mathrm{~K}^{-1}$ \\
\hline Hardening/softening & $\omega_{h}=2.0 \times 10^{-3} \mathrm{~K}^{-1}$ \\
\hline Reference temperature & $T_{\text {ref }}=293 \mathrm{~K}$ \\
\hline
\end{tabular}

As mentioned previously, initially the tests were performed considering a constant time step $(\Delta t=0.2 \mathrm{~s})$, corresponding to a total of 11 increments. The relative temperature evolution predicted by each coupling algorithm is presented in Figure 6, while the evolution of the equivalent (von Mises) stress is shown in Figure 7. The temperature increases during the loading due to the heat generated by plastic deformation. On the other hand, the von Mises stress decreases after the first two increments because the flow stress decreases with the temperature (softening). The norm of the relative error of the TMMT and MMT solutions is also presented in the same figures. The error was evaluated considering the iterative solution as reference (MMIT), since this is the most accurate.

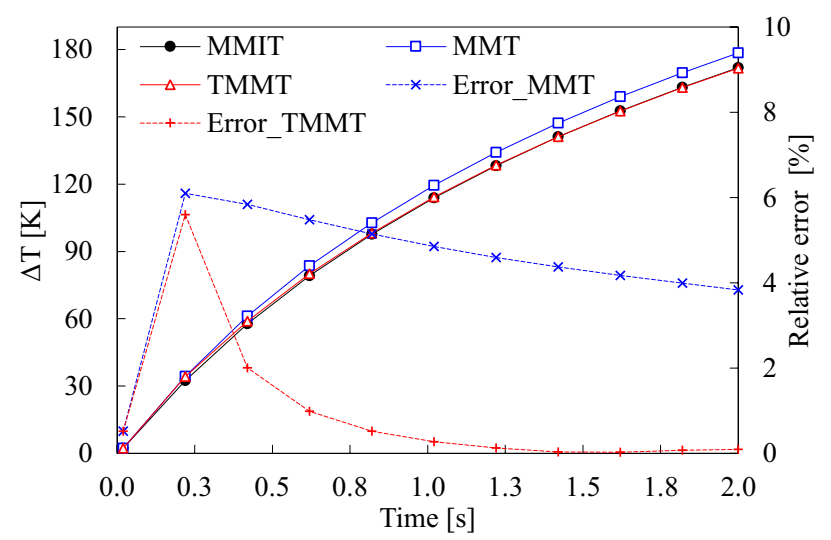

Figure 6. Relative temperature evolution $(\Delta \mathrm{T}=\mathrm{Ti}$-Tref $)$ obtained with the TMMT, MMT and MMIT algorithms, including the relative error calculated for the TMMT and MMT solutions (single element tensile test with constant time step). 


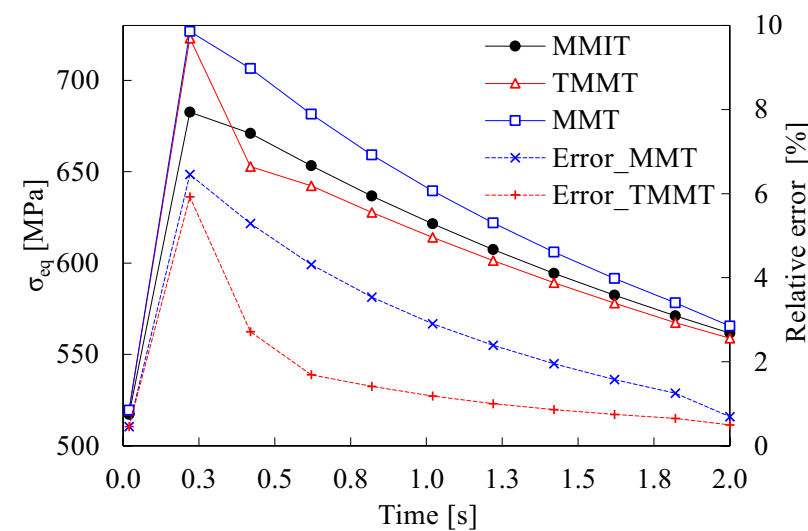

Figure 7. Equivalent (von Mises) stress evolution obtained with the TMMT, MMT and MMIT algorithms, including the relative error calculated for the TMMT and MMT solutions (single element tensile test with constant time step).

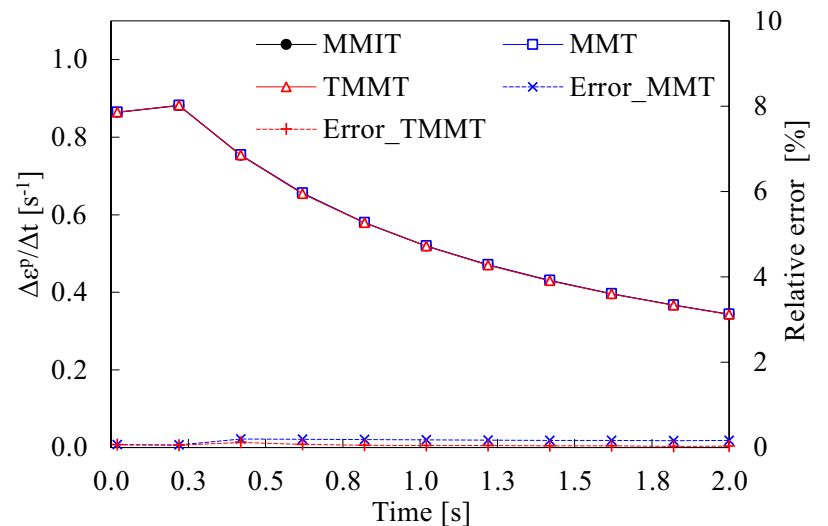

Figure 8. Plastic strain rate obtained with TMMT, MMT and MMIT algorithms, including the error calculated for the TMMT and MMT solutions (single element tensile test with constant time step).

For the first increment, the error of the algorithms TMMT and MMT is identical because there is no history of plastic deformation. Indeed, the TMMT algorithm uses the value of the plastic heat generated in the previous increment to predict the trial temperature field. The error of both algorithms attains the maximum value in the second increment, as shown in Figure 6 and Figure 7. This error is directly related with the temperature increment. Since the magnitude of the temperature increment is connected with the amount of heat generated by plastic deformation, the evolution of the plastic strain rate is presented in Figure 8, for both algorithms. For this variable the difference between coupling algorithms is negligible, because the increment of plastic strain is mainly dictated by the finite element deformed configuration, which is imposed by the prescribed displacement. Therefore, the maximum temperature increment occurs in the second increment, since both the equivalent stress (Figure 7) as the increment of plastic strain (Figure 8) attain their maximum values. However, this temperature increment is not taken into account by the MMT algorithm to induce the mechanical softening effect during this increment, leading to an overestimation of the von Mises stress. On the other hand, the prediction phase of the TMMT algorithm, which is used to generate a temperature field for the actual increment based on the last increment, provides a poor temperature estimative for the second increment. This results from the very small value of temperature increment in the first increment (a small amount of heat generated by plastic deformation). Nevertheless, after the second increment the error of the TMMT algorithm is significantly reduced, as shown in Figure 6 and Figure 7.

During the test, the equivalent stress and the rate of plastic strain show a decreasing trend. Thus, the temperature increment also decreases in each increment. Accordingly, the prediction phase of the TMMT algorithm overestimates the temperature field, which originates an underestimation of the equivalent stress, as shown in Figure 7. Since the MMT algorithm solves the mechanical field based on the last temperature field, equivalent stress is overestimated.

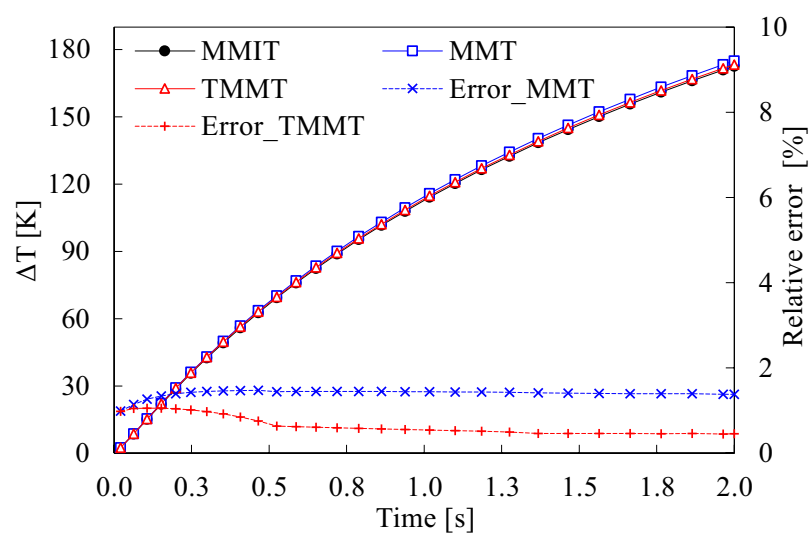

Figure 9. Relative temperature evolution $(\Delta \mathrm{T}=\mathrm{Ti}$-Tref $)$ obtained with the TMMT, MMT and MMIT algorithms and relative error calculated for the TMMT and MMT solutions (single element tensile test with the $r_{\text {min }}$ strategy activated).

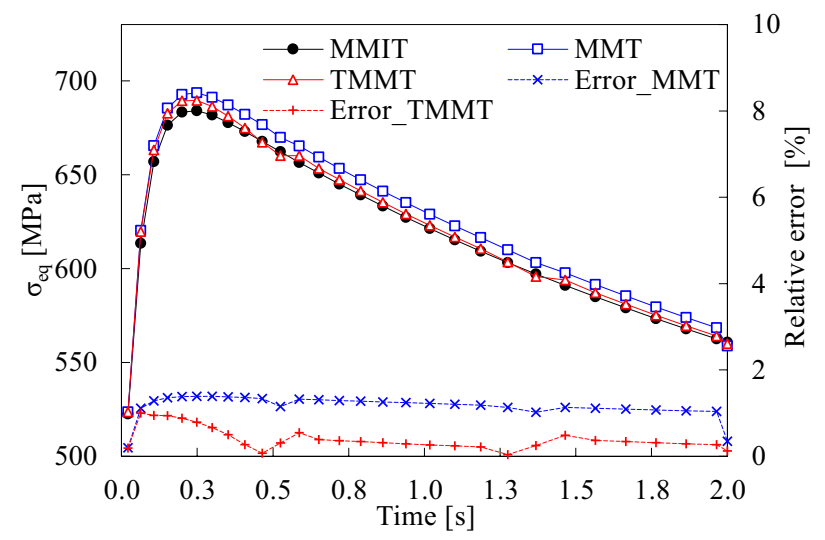

Figure 10 Equivalent von Mises stress evolution obtained with the TMMT, MMT and MMIT algorithms and relative error calculated for the TMMT and MMT solutions (single element tensile test, with the $r_{\text {min }}$ strategy activated).

In order to assess the influence of the increment size in the accuracy of the coupling algorithms presented, the $r_{\min }$ strategy was activated. The obtained results for the evolutions of the relative temperature, the von Mises stress and the rate of plastic strain are shown in Figure 9, Figure 10 and Figure 11, respectively. The introduction of the $r_{\text {min }}$ strategy to adjust the time step leads to a global decrease of the step size. Indeed, the number of increments increased from 11 to 29 . Consequently, the accuracy of all coupling algorithms is improved, as shown in Figure 9 and 
Figure 10. The relative error of the solution predicted by the TMMT algorithm is globally lower than half the one obtained with the MMT algorithm. Therefore, using the same increment size, the proposed coupling algorithm provides results more accurate than the ones obtained with the classical staggered strategy (MMT).

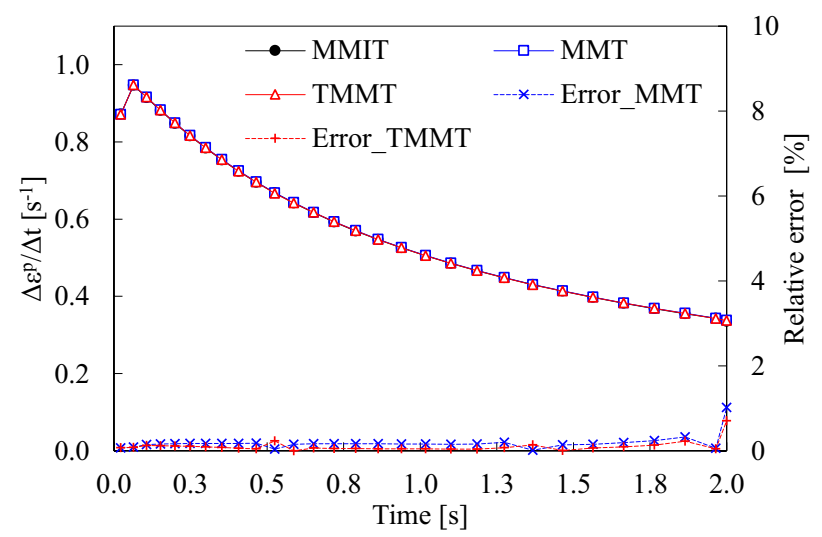

Figure 11. Rate of plastic deformation obtained with TMMT, MMT and MMIT algorithms, including the error calculated for the TMMT and MMT solutions (single element tensile test, with the $r_{\min }$ strategy activated).

\section{Conclusion}

The present study aims to improve the accuracy of thermomechanical simulations, within a reasonable computational cost. In this context, a new staggered coupling strategy was proposed, which involves a predictor and a correction phase, in which both the thermal and the mechanical problem are solved sequentially. In the predictor phase the thermal problem is solved previous to the mechanical, while in the correction phase the sequence is inverted. The finite element code developed was validated using a classical thermomechanical problem. The performance of the proposed strategy, in terms of accuracy, was discussed and compared with the one of classical strategies. The results achieved for a problem involving large thermo-elasto-plastic deformations and rotations show an improvement in the accuracy, when compared with the classical staggered algorithm. However, more complex examples need to be used to enable the assessment of the computational efficiency.

\section{Acknowledgements}

The authors gratefully acknowledge the financial support of the Portuguese Foundation for Science and Technology (FCT) under projects with reference UID/EMS/00285/2013, PTDC/EMS-TEC/0702/2014 (POCI-01-0145-FEDER-016779) and PTDC/EMSTEC/6400/2014 (POCI-01-0145-FEDER-016876) by UE/FEDER through the program COMPETE2020. The second author is also grateful to the FCT for the Postdoctoral grant SFRH/BPD/101334/2014.

\section{References}

1. S. Rothe, P. Erbts, A. Düster, S. Hartmann, Comput.
Methods Appl. Mech. Eng. 293 (2015) 375-410.

2. C.A. Felippa, K.C. Park, C. Farhat, Comput. Methods Appl. Mech. Eng. 190 (2001) 3247-3270.

3. F. Armero, J.C. Simo, Int. J. Plast. 9 (1993) 749-782.

4. M. Vaz, P.A. Muñoz-Rojas, M.R. Lange, Int. J. Mech. Sci. 53 (2011) 387-398.

5. T. Netz, S. Hartmann, Comput. Math. with Appl. 70 (2015) 1457-1480.

6. V. Dunić, N. Busarac, V. Slavković, B. Rosić, R. Niekamp, H. Matthies, R. Slavković, M. Živković, Contin. Mech. Thermodyn. (2015).

7. L. Adam, J. Ponthot, J. Eng. Mech. 128 (2002) 1222-1232.

8. M. Canadija, J. Brnic, Int. J. Plast. 20 (2004) 18511874.

9. H.L. Xing, a. Makinouchi, Eng. Comput. 19 (2002) $392-410$.

10. L. Adam, J.P. Ponthot, Int. J. Solids Struct. 42 (2005) 5615-5655.

11. F. Armero, J. C. Simo, Int. J. Numer. Methods Eng. 35 (1992) 737-766.

12. C. Agelet De Saracibar, M. Cervera, M. Chiumenti, Int. J. Plast. 15 (1999) 1-34.

13. A. Ibrahimbegovic, L. Chorfi, Int. J. Solids Struct. 39 (2002) 499-528.

14. M.H. Ulz, Comput. Methods Appl. Mech. Eng. 198 (2009) 3262-3277.

15. L.F. Menezes, C. Teodosiu, J. Mater. Process. Technol. 97 (2000) 100-106.

16. M.C. Oliveira, J.L. Alves, B.M. Chaparro, L.F. Menezes, Int. J. Plast. 23 (2007) 516-543.

17. Y. Yamada, N. Yoshimura, T. Sakurai, Int. J. Mech. Sci. 10 (1968) 343-354.

18. M.C. Oliveira, L.F. Menezes, Finite Elem. Anal. Des. 40 (2004) 1995-2010.

19. D.M. Neto, M.C. Oliveira, L.F. Menezes, J.L. Alves, Comput. Methods Appl. Mech. Eng. 299 (2015) 283-315.

20. L. Adam, J.-P. Ponthot, J. Eng. Mech. 128 (2002) $1222-1232$.

21. A. Andrade-Campos, F. da Silva, F. Teixeira-Dias, Int. J. Numer. Methods Eng. 70 (2007) 582-609.

22. J. Hodowany, G. Ravichandran, a Rosakis, P. Rosakis, Exp. Mech. 40 (2000) 113-123.

23. G.I. Taylor, H. Quinney, Proc. R. Soc. A Math. Phys. Eng. Sci. 143 (1934) 307-326.

24. J.M.P. Martins, J.L. Alves, D.M. Neto, M.C. Oliveira, L.F. Menezes, Int. J. Adv. Manuf. Technol. (2015).

25. D. Pantuso, K.-J. Bathe, P.A. Bouzinov, Comput. Struct. 75 (2000) 551-573.

26. K.J. Bathe, Finite Element Procedures, Prentice-Hall: Englewood Cliffs, 1996.

27. A. Ibrahimbegovic, (2009).

28. J.H. Argyris, J.S. Doltsinis, Comput. Methods Appl. Mech. Eng. 25 (1981) 195-253.

29. J.C. Simo, C. Miehe, Comput. Methods Appl. Mech. Eng. 98 (1992) 41-104. 